\title{
Modified osteo-odonto-keratoprosthesis (MOOKP) surgery: case report, one year of follow up
}

\begin{abstract}
Osteo-Odonto-Keratoprosthesis (OOKP) is a surgical technique created by Dr. Strampeli in 1964 and modified by Dr. Falcinelli (MOOKP). The technique is described in detail in the Rome protocol - Viena. The MOOKP involves an autologous transplant, in which an osteo-dental lamina is used (osteo-odonto lamina), extracted from the maxillary, like biological support for a polymethyl methacrylate (PMMA) cylinder. The case report corresponds to the first patient operated of MOOKP in America, which was performed in the MOOKP Center - Latin America in the Clinical Hospital of the University of Chile, in Santiago, Chile.
\end{abstract}

Keywords: Cornea, blindness, tooth, prosthesis, mookp, ookp, kpro, keratoprosthesis, patient, surgical technique, cornea transplants, injury, osteo-odonto lamina
Volume 2 Issue 4 - 2018

\author{
Christian Fau, Solange Nabzo \\ Fundación Oftalmológica 2020, Iberoamerican Cochrane \\ Network, Santiago, Chile
}

\begin{abstract}
Correspondence: Christian Fau, Fundación Oftalmológica 2020, Avd. Presidente Riesgo 5157, Dep. 212, Las Condes, Santiago, Metropolitana, Chile, Zip Code 7560854, Tel +5628216334, Email chfauf@gmail.com, cfau@fundacion2020.org t
\end{abstract}

Received: July 30, 2018 | Published: August 31, 2018

\section{Introduction}

The Keratoprosthesis (KPRO) exists to be used in cases of patients where the cornea transplants or other surgical techniques do not obtain good results; in these patients who are blind or have had serious injury the KPRO offers nowadays the only possible alternative. OsteoOdonto-Keratoprosthesis (OOKP) is a surgical technique created by Dr. Strampeli in 1964 and modified by Dr. Falcinelli (MOOKP). ${ }^{1,2}$ The technique that we used on this patient was MOOKP, which is described in detail in the Rome protocol - Viena, ${ }^{2}$ this is a proven and standardized technique, although there are some variations to this technique published by other authors, these have not demonstrated to be better than the classic technique and they could have a higher rate of complications. The MOOKP involves an autologous transplant, in which an osteo-dental lamina is used (osteo-odonto lamina), extracted from the maxillary, like biological support for a polymethyl methacrylate (PMMA) cylinder, which after a period from 2 to 3 months of integration and vascularisation under the skin, it is placed on the corneal surface of an ocular globe previously prepared for this purpose, allowing light passing through and therefore recovering the vision, taking the patients from your state of blindness and allowing them to have a normal life. ${ }^{2,3}$ The case report corresponds to the first patient operated of MOOKP in America, which was performed in the MOOKP Center - Latin America in the Clinical Hospital of the University of Chile, in Santiago, Chile, performed by Professor doctor GianCarlo Falcinelli and doctor Giovanni Falcinelli, assisted by specialist doctors in cornea, retina, glaucoma, radiology, maxillofacial surgery and anesthesia.

\section{Case report}

A 32 year old male patient without medical antecedents of importance who worked as an operator in a steam boiler room in a food industry. In the year 2004, while he was working the boiler exploded, thus he suffered severe burns all over his body, including both eyes. The corporal burns, product of the injury, required multiple skin surgical grafts along with the eyelids of both eyes. The corneas of both eyes presented severe burns by hot water, despite the treatment used initially, evolved to the total opacity and in addition developed a posterior vascularisation; the patient was left with the vision of light and with good projection (Figure 1). During the following months and years the patient was treated with total corneal transplants plus tectonic graft of esclero-corneal limbus in 3 opportunities, during the post operating of the right eye, product of a secondary glaucoma, it required an Amhed valve implant, the patient's outcome was bad, product of a great vascularisation produced by the accident, all the transplants were rejected, the patient remains only with light vision. In May 2008, the patient was evaluated for the first time in the Clinical Hospital of the University of Chile, after this it was decided to implant MOOKP in the left eye since this eye did not have glaucoma antecedents. An echobiometry was made into the left eye that revealed a normal posterior camera, with applied retina in $360^{\circ}$, total posterior vitreous detachment and normal papilla excavation. On May 15th 2008, the patient was operated in the Clinical Hospital of the University of Chile by Professor doctor GianCarlo Facinelli and doctor Giovani Falcinelli plus a multidisciplinary equipment of the Clinical Hospital, where it was made the first surgical time, of a surgical plan of 3 times. $^{4-6}$ In this first surgical procedure the following was performed:

I. Partial laminar Keratectomy on the left eye

II. Iridectomy in 360 degrees

III. Intra-capsular Phakoeresis

IV. Vitrectomy

V. Conjuntival covering

VI. Left upper canine dento-alveolar Osteotomy

VII. Lamina Carving

VIII.Lens implant in osteo- odonto keratoprosthesis plate (OOKP) (Figure 2)

IX. OOKP plate implant in the subcutaneous pocket below the right lower eyelid. (Figure 1) \& (Figure 3)

On June20th 2008, the second surgical procedure was performed on the patient; it was made a partial laminar Keratectomy on the left eye plus an ocular surface covering of the left eye with buccal mucosal graft. Finally, on August 26th 2008, the third surgical procedure was completed with the OOKP plate implant on the left eye plus an anterior vitrectomy (Figure 4). In all the surgeries the patient 
had a satisfactory outcome, without presenting complications, in all of them were used the following prophylactic scheme: clindamycin plus intravenous ciprofloxacin, topical ocular antibiotics of gatifloxacin and tobramycin, with a good intraocular pressure control digitally measured, with topical medicines: Travatan, Timoptic, Brimonidine, Trusopt and oral Diamox. The patient product of a trichiasis of the eyelashes of the lower eyelids of both eyes, produced by initial face burns, developed an inflammation of the mucosa caused by the eyelashes rubbing, from what it required an upper palpebral margin rotation surgery on both eyes, it was performed on October 16,2008 . The only post surgery complication that has appeared until Table I Details of Visual Acuity Postoperative the moment in the patient has been the buccal mucosal growth by over the cylinder, lowering the view field; therefore the mucosa had to be trimmed on two occasions (Figure 5). ${ }^{7}$ Visual evaluation of the operated eye of the patient shows in Table 1, where the great visual improvement that the patient has experienced, from light vision with good projection to $20 / 20$ with a $+0,75$ sph correction, an excellent anatomical and functional success, leaving the 4 years of blindness condition, standardizing his/her familiar and personal activities. At the moment the patient is under the control and treatment with medicines to prevent glaucoma, in rehabilitation of his dental health..$^{8-14}$

\begin{tabular}{llllll}
\hline & Before & $\mathbf{4 8}$ hrs. After & I Week & I Month & $\mathbf{3}$ Month \\
\hline Visual Acuity Far & Light with projection & Recognize faces & $0,5 \circ 20 / 40$ & $0,67 \circ 20 / 30$ & $0,67 \circ 20 / 30$ \\
Corrección & - & - & $+3.00 \mathrm{sph}$ & $'+1.5 \mathrm{sph}$ & $+0.75 \mathrm{sph}$ \\
Visual Acuity near & - & - & - & - & Jaeger 7 \\
Corrección & - & - & - & - & + \\
\hline
\end{tabular}

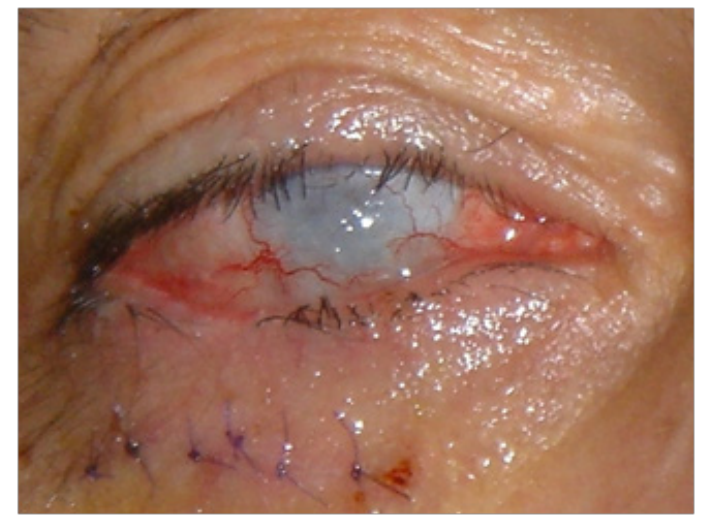

Figure I The corneas of both eyes presented severe burns by hot water, with the total opacity and in addition vascularisation. OOKP plate implant in the subcutaneous pocket below the right lower eyelid.

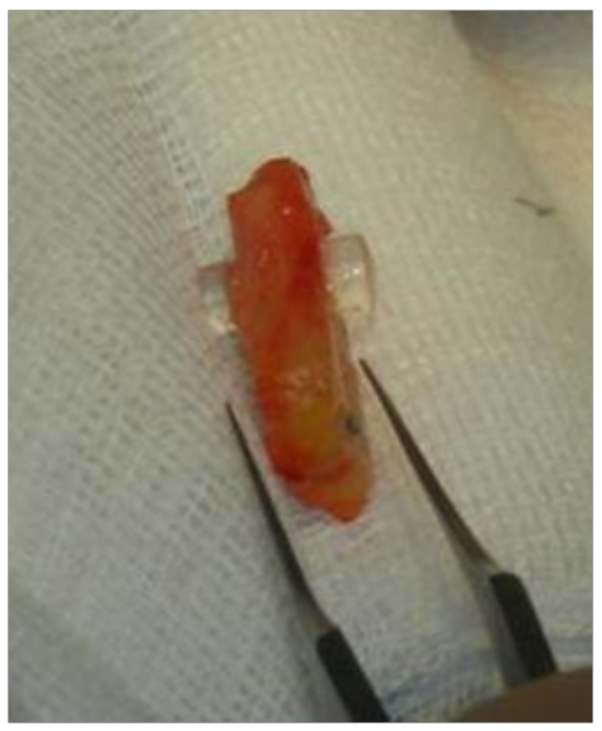

Figure 2 Lens implant in osteo- odonto keratoprosthesis plate.

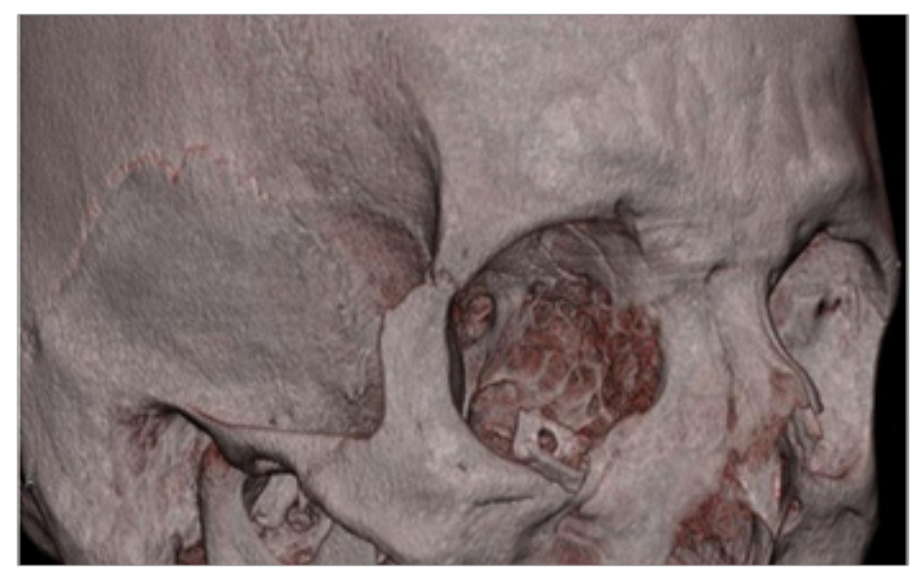

Figure 3 OOKP plate implant in the subcutaneous pocket below the right lower eyelid.

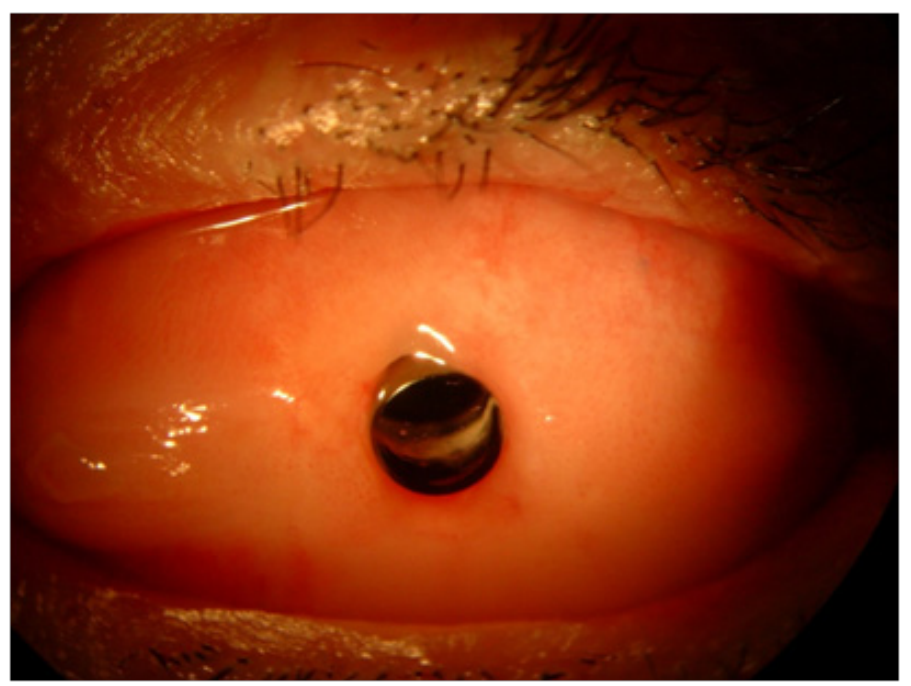

Figure 4 Modified Osteo-Odonto-Keratoprosthesis (MOOKP) Surgery. 


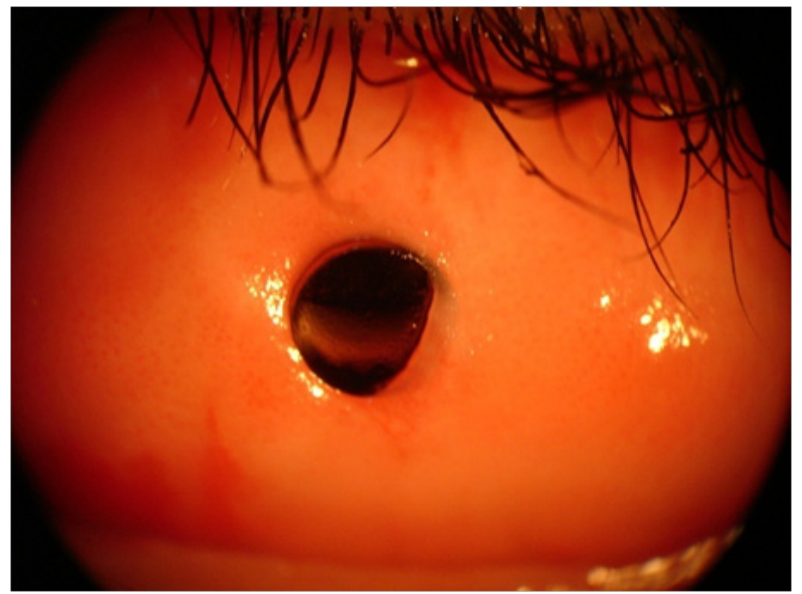

Figure 5 Modified Osteo-Odonto-Keratoprosthesis Surgery with buccal mucosal growth.

\section{Discussion}

The OOKP technique (osteo- odonto keratoprosthesis) was developed by the Dr. Benedetto Strampelli more than 35 years ago, ${ }^{1}$ later was perfected by modifications made by the Professor Dr. GianCarlo Falcinelli, technique that is recognized and accepted throughout the world, and that at the moment is certified by the "Rome-Vienna" protocol published in "Cornea" magazine in 2005. ${ }^{2}$ The surgical technique is of great complexity, requires the accomplishment of 3 surgeries on the patient, each of them being very laborious, the accomplishment of them requires a great training and until now it had not been possible to perform it in Chile nor in America. The technique suitably performed allows achieving an anatomical success of $98 \%$ and a visual functional success of $88 \%{ }^{3-8}$ The KPRO has a great patient follow-up reported, near 35 years, unlike other keratoprosthesis that are of transitory function, this allows not only the vision recovery but the maintenance of this condition throughout the time. In blind selected patient that are not treatable by other surgical techniques the MOOKP is a safe and effective surgery in recovering the vision of these patients and it would have to be the surgery of first election. The objective of the project is to take care of national and international patients who are candidates for this surgery, and to become a training center where American specialist will be able to be trained in order to spread this surgical technique. This collaboration project was developed with the effort of the Institute of OOKP of Rome, the Italo - latinamerican Institute, Italian Embassy, DECYTI, Ministry of Foreign Affairs of Chile, University of Chile and 2020 Ophthalmological Foundation.

\section{Funding details}

There is no funding from other institutions for this study.

\section{Acknowledgements}

Thanks to Professor Doctor GianCarlo Falcinelli and Doctor Giovanni Falcinelli, for his great and disinterested contribution to the improvement of ophthalmology in Chile.

\section{Conflict of interests}

Authors declare that there is no conflict of interest.

\section{References}

1. Strampelli B. Osteo-odonto-keratoprotesi. Anales Inst Baraquer. 1974;12:21.

2. Hille K, Grabner G, Liu C, et al. Standards for Modified Osteoodontokeratoprosthesis (OOKP) Surgery According to Strampelli and Falcinelli: The Rome-Vienna Protocol. Cornea. 2005;24(8):895-908.

3. Falcinelli G, Falsini B, Taloni M, et al. Modified osteo-odontokeratoprosthesis for treatment of corneal blindness: long-term anatomical and functional outcomes in 181 cases. Arch Ophthalmol. 2005;123(10):1319-29.

4. Ralph M, Charoenrook V, De la Paz M, et al. Long-term functional and anatomical results of osteo- and osteoodonto-keratoprosthesis. Graefes Archive for Clinical \& Experimental Ophthalmology. 2008;246(8):11331137.

5. Tan A, Tan DT, Tan X-W, et al. Osteo-odonto keratoprosthesis: systematic review of surgical outcomes and complication rates. Ocul Surf. 2012;10(1):15-25.

6. Iyer G, Pillai VS, Srinivasan B, et al. Modified osteo-odonto keratoprosthesis--the Indian experience--results of the first 50 cases. Cornea. 2010;29(7):771-776.

7. Avadhanam VS, Herold J, Thorp S. Mitomycin-C for mucous membrane overgrowth in OOKP eyes. Cornea. 2014;33(9):981-984.

8. Rishi P, Rishi E, Agarwal V. Vitreoretinal Complications and Outcomes in 92 Eyes Undergoing Surgery for Modified Osteo-Odonto-Keratoprosthesis: A 10-Year Review. Ophthalmology. 2018;125(6):832-841.

9. Hille K, Hille A, Ruprecht KW. Medium term results in keratoprostheses with biocompatible and biological haptic. Graefes Archive for Clinical \& Experimental Ophthalmology. 2006;244(6):696-704.

10. Hille K, Landau H, Ruprecht KW. Die Osteo-Odonto-Keratoprothese. Ein Resumee nach sechs Jahren operativer Erfahrung. [Osteoodonto-keratoprosthesis. A summary of 6 years surgical experience]. Ophthalmologe. 2002;99(2):90-95.

11. Falcinelli G, Falsini B, Taloni M, et al. Detection of glaucomatous damage in patients with osteo-odontokeratoprosthesis. British Journal of Ophthalmology. 1995;79(2):129-134.

12. Marchi V, Ricci R, Pecorella I. Osteo-odonto-keratoprosthesis: description of surgical technique with results in 85 patients. Cornea 1994;13:125-30.

13. Alvarez de Toledo J, Barraquer RI, Temprano J. Osteo-odonto keratoprosthesis: a 30 years retrospective study. An Inst Barraquer. 1999;28(Suppl 1):95-100.

14. Sciscio A, Herold J, Hull C. Early British results of modern Falcinelli osteo-odonto-keratoprosthesis (OOKP) surgery. An Inst Barraquer. 2001;30:59-63. 